\title{
Magnetic resonance imaging findings in a case of congenital rubella encephalitis
}

\author{
Vijay Sawlani MD FRCR EQNR MSC ${ }^{1}$, Jai Jai Shiva Shankar MD DM MSC ${ }^{2}$, Cathy White MD FRCP1
}

$\mathrm{R}$ ubella is an extremely rare disease in the Western world because it has been nearly eliminated since routine rubella immunization was introduced. The likely outcome of congenital infection is related to the timing of infection during gestation. Rubella acquired by the mother in early pregnancy can have devastating effects on unborn children. Congenital rubella syndrome (CRS) can rarely present with a progressive rubella panencephalitis (PRP) or subacute sclerosing panencephalitis (SSPE) (1). We present magnetic resonance imaging (MRI) findings in a case of serology-positive congenital rubella encephalitis.

\section{CASE PRESENTATION}

A seven-year-old girl, who had been born at 34 weeks' gestation by emergency Caesarean section due to poor fetal growth, was diagnosed with congenital rubella at two weeks of age. Her birth weight was $1790 \mathrm{~g}$ (25th percentile). Her prenatal ultrasound examination was unremarkable with the exception of oligohydramnios with symmetric growth retardation below the fifth percentile observed in the third trimester. She remained in the neonatal intensive care unit for two weeks because of feeding difficulty and difficulty in maintaining her temperature, and she developed a rash.

Four days after birth, screening for toxoplasma, rubella, cytomegalovirus and herpes virus revealed an immunoglobulin $\mathrm{M}$ antibody test positive for rubella, consistent with intrauterine rubella infection. Her mother had no clinical illness suggestive of rubella infection and had been vaccinated against rubella before the pregnancy. However, her rubella radial hemolysis test at seven weeks of pregnancy showed antibody (immunoglobulin $\mathrm{G}$ ) titre $>15 \mathrm{IU} / \mathrm{mL}$ and a positive rubella hemagglutination inhibition test $(1 / 1024)$. A repeat test seven months later confirmed antibody titre $>15 \mathrm{IU} / \mathrm{mL}$. This was suggestive as intermediately reactive for rubella. The rubella immunoglobulin $\mathrm{M}$ test was negative. These results were suggestive of rubella reinfection. The reinfection of rubella virus occurs in mothers with antibody to rubella virus during pregnancy and may eventually result in CRS in the baby. Rapid plasma reagin test and Treponema pallidium hemagglutination test was negative.

The child was found to be profoundly deaf and her initial development was delayed. On examination at seven years of age, her head circumference was small for her age, but she was not microcephalic. She developed signs of precocious puberty, likely due to late-onset endocrine disturbances of an autoimmune etiology. She had a minimal right convergent squint and no other cranial nerve abnormality. She had a spastic diplegia and severe learning difficulties. These abnormalities were within the spectrum of abnormalities found in children with CRS. However, there was no history of regression of milestones.

An MRI of the brain performed at seven years of age showed multiple focal periventricular and subcortical fluid-attenated inversionrecovery hyperintense lesions suggestive of gliosis or demyelinating lesions (Figure $1 \mathrm{E}$ and $1 \mathrm{~F}$ ). There were several cystic changes in bilateral temporal lobes (Figure 1C, 1D and 1E), more prominent toward the right side, suggestive of encephalomalacia. There were multiple foci of calcified nodules in the periventricular region on plain computed tomography scanning and gradient echo sequence (Figure 1A and 1B). There was no evidence of diffuse brain atrophy.

\section{DISCUSSION}

Rubella, or 'German measles', is caused by rubella virus. The incidence of encephalitis is one in 6000 cases of rubella. Rubella is generally a mild disease that causes a rash and fever for two to three days. The symptoms are often mild and may be missed in 30\% to 50\% of cases. Three distinct syndromes following rubella virus infection have been described in the literature $(1,2)$ : postinfectious encephalitis following acute infection, with unknown pathogenesis; congenital infection, which causes a spectrum of neurological features due to viral invasion and replication in the brain; and PRP, a rare immune-mediated neurodegenerative disorder occuring after congenital or postnatal infection.

The outcome of infection during pregnancy is related to the gestational age at the time of infection $(1,2)$. If a fetus is infected before the 20 th week of gestation, CRS can occur. In the first 10 weeks of gestation, maternal infection leads to fetal damage in $90 \%$ of infants, with multiple defects. The congenital malformation syndrome includes ventricular and atrial septal defects, cataracts, glaucoma, mental and motor retardation, and hearing difficulty $(1,2)$. At 16 weeks' gestation, the risk of damage decreases to $10 \%$ to $20 \%$, with only minimal risk of deafness. After 20 weeks' gestation, there is no documented risk. The congenital infection can also lead to premature delivery or fetal death $(1,2)$.

PRP is a rare neurodegenerative disorder and an uncommon late complication of CRS $(1,2)$. Its pathogenesis is similar to SSPE and progressive multifocal leukoencephalopathy, caused by measles virus and papovavirus, respectively. All three disorders are characterised by slow mental and motor deterioration (1-3).



Figure 1) Multiple periventricular calcified nodules (small arrow) on computed tomography scan (A) and gradient echo (B) axial images. Right temporal cystic changes (bold arrow) seen on axial T1-weighted (C), T2-weighted (D) and coronal fluid-attenated inversion-recovery (E) images, with multiple areas of periventricular demyelination or gliosis seen on coronal fluid-attenated inversion-recovery images (F) suggestive of sequelae of congenital rubella infection

${ }^{1}$ Morriston Hospital, Abertawe Bro Morgannwg University National Health Service Trust, Swansea, United Kingdom; ${ }^{2}$ Department of Diagnostic Imaging, Neuroradiology Section, Queen Elizabeth II Hospital, Halifax, Nova Scotia

Correspondence: Dr Jai Jai Shiva Shankar, Department of Diagnostic Imaging, Neuroradiology Section, Queen Elizabeth II Hospital, Halifax,

Nova Scotia B3H 1E6 Telephone 902-489-0577, fax 902-473-6235, e-mail shivajai1@gmail.com, shivajai1@rediffmail.com 
The prominent pathological findings in the brain in PRP include diffuse destruction of white matter, with perivascular inflammatory cells and gliosis with moderate neuronal loss, numerous amorphous vascular deposits in the white matter and severe generalized cerebellar atrophy $(4,5)$. Similar changes are observed in congenital rubella and other latent viral illnesses $(4,5)$.

The MRI findings of gliosis, demyelinating lesions and periventricular calcification may represent the sequelae of rubella infection and are reported in the pathology literature $(4,5)$. The atrophic changes in temporal lobes observed in our patient, possibly secondary to encephalitis or progressive panencephalitis, have not been described previously in cases of congenital rubella infection. However, the affinity of rubella virus or tissue tropism toward the temporal lobe is not known and is likely an anecdotal finding in our patient.

Abe and Date (6) have categorized the nervous system disorders caused by rubella virus infection as acute and chronic. The former consists of acute encephalitis and peripheral neuropathy, and the latter CRS and PRP. The incidence of acute rubella encephalitis is one in 6000 rubella patients; this presents as either viremia or eruption stage, which is a demyelinating disease designated as secondary encephalitis $(7,8)$. The prognosis of this disease is not severe, with recovery without any sequelae in $80 \%$ of cases $(6,7)$.

CRS is a congenital malformation syndrome that includes cataracts, glaucoma, ventricular and atrial septal defects, mental and motor retardation, and hearing difficulty (6). The incidence of intrauterine rubella as a cause of deafness is likely underestimated (9). The reinfection by rubella virus during pregnancy in a mother with antibodies to rubella has also been reported by Abe and Date (6) and is likely apparent in our case. The mechanisms responsible for maternal-fetal

\section{REFERENCES}

1. Wolinsky JS. Progressive rubella panencephalitis. In: RR McKendall, ed. Handbook of Clinical Neurology, Volume

12(56): Viral diseases. Elsevier Science Publishers, 1989:405-412.

2. Frey TK. Neurological aspects of rubella virus infection. Intervirology 1997;40:167-75.

3. Kuroda Y, Matsui M. Progressive rubella panencephalitis. Nippon Rinsho 1997;55:922-5.

4. Townsend JJ, Wolinsky JS, Baringer JR. The neuropathology of progressive rubella panencephalitis of late onset. Brain 1976;99:81-90.

5. Towensend JJ, Stroop WG, Baringer JR, et al. Neuropathology of progressive rubella panencephalitis after childhood rubella. Neurology 1982;32:185-90.

6. Abe T, Date M. Rubella virus. Nippon Rinsho 1997;55:865-9.

7. American Academy of Pediatrics: 2000 Red Book: Report of the Committee on Infectious Diseases, 25th edn. American Academy of Pediatrics; 2000.

8. Cherry JD. Rubella virus. In: Feigin RD, Cherry JD, eds. Textbook of Pediatric Infectious Diseases, Volume 2. Philadephia: WB Saunders Company, 1998:1922-49. transmission in women with pre-existing rubella antibodies are, however, unclear (10).

In children with congenital rubella infection, the deficits remain stable and the neurological deterioration is not believed to occur after the first few years of life (11). However, chronic progressive panencephalitis is a chronic progressive neurological illness characterised by slow mental and motor deterioration $(11,12)$ and may simulate SSPE (12). Weil et al (12) reported a case involving a 12 -year-old boy with CRS in whom rubella virus was isolated from the patient's brain. On microscopy, the brain tissue showed panencephalitis similar to SSPE.

The MRI findings of demyelination or gliosis, periventricular calcification and bilateral temporal lobe atrophy may represent the sequlae of rubella infection in our case. The other possibility is leukoencephalopathy with bilateral temporal lobe cysts (13). Our patient had a relatively small head circumference, although this did not meet the definition of microcephaly. In view of the presence of positive rubella serology and other findings, such as periventricular calcifications, this is most likely the sequelae of congenital rubella infection. At present, the neurological status of the patient is stable; however, the disease may progress to PRP, as suggested by the atrophic changes in temporal lobes on MRI.

\section{CONCLUSION}

In an appropriate clinical setting, the MRI findings of gliosis, demyelination, periventricular calcification and cystic atrophic changes in temporal lobes may represent sequelae of rubella infection secondary to rubella encephalitis or progressive panencephalitis.

9. Iuorio JL, Hosking CS, Pyman C. Retrospective diagnosis of congenital rubella. Br Med J (Clin Res Ed) 1984;289:1566-8.

10. O'Shea S, Corbett KM, Barrow SM, Banatvala JE, Best JM. Rubella reinfection; role of neutralizing antibodies and cell mediated immunity. Clin Diagn Virol 1994;2:349-58.

11. Towsend JJ, Baringer JR, Wolinsky JS, et al. Progressive rubella pan encephalitis. Late onset after congenital rubella. N Engl J Med 1975;292:990-3.

12. Weil ML, Itabashi $\mathrm{H}$, Cremer NE, et al. Chronic progressive panencephalitis due to rubella virus simulating subacute sclerosing panencephalitis. N Engl J Med 1975;292:994-8.

13. Battini R, Bianchi MC, Tosetti M, Guzzetta A, Cioni G. Leukoencephalopathy with bilateral anterior temporal lobe cysts: A further case of this new entity. J Child Neurol 2002;17:773-6. 


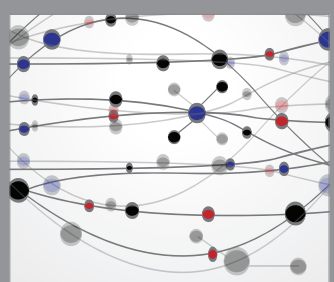

The Scientific World Journal
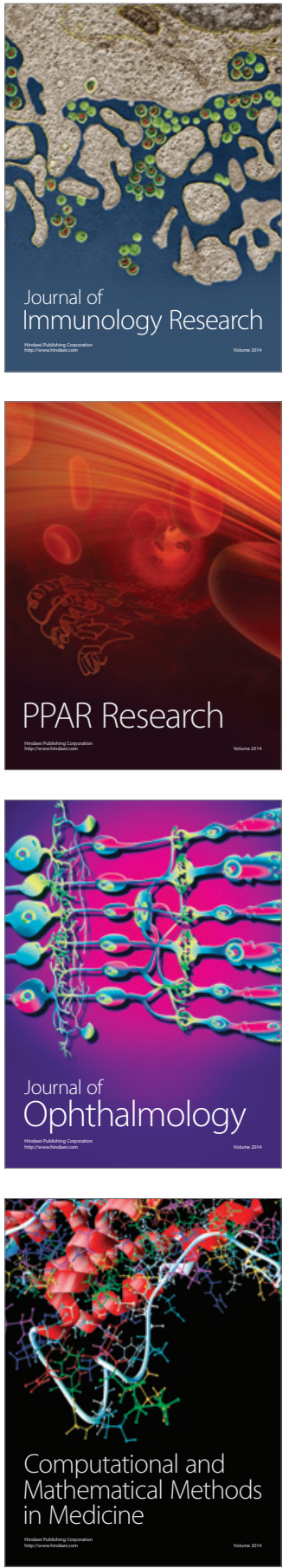



Gastroenterology Research and Practice

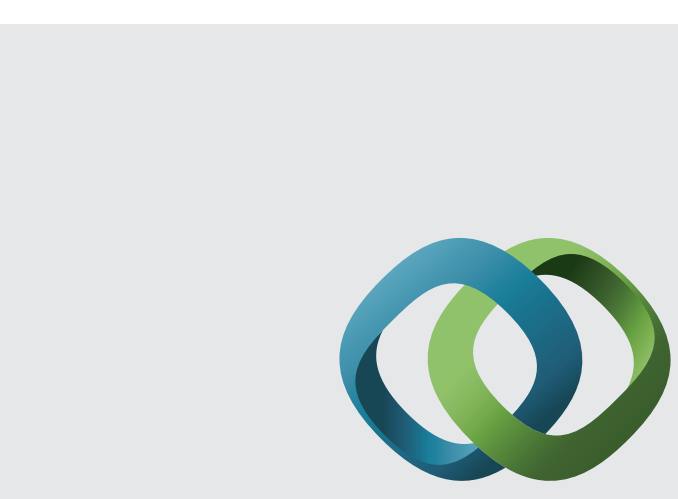

\section{Hindawi}

Submit your manuscripts at

http://www.hindawi.com
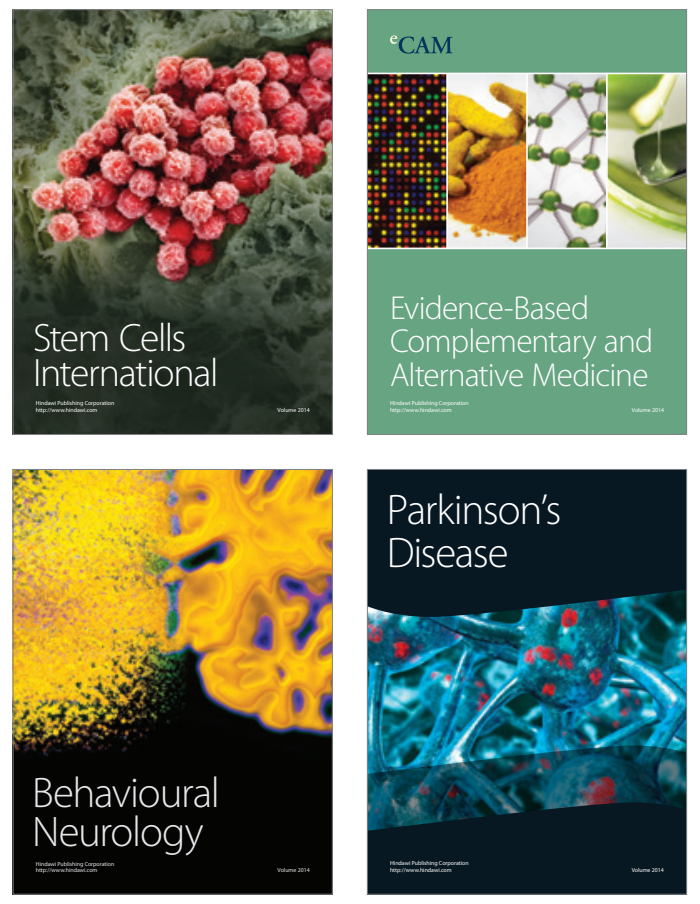


Disease Markers
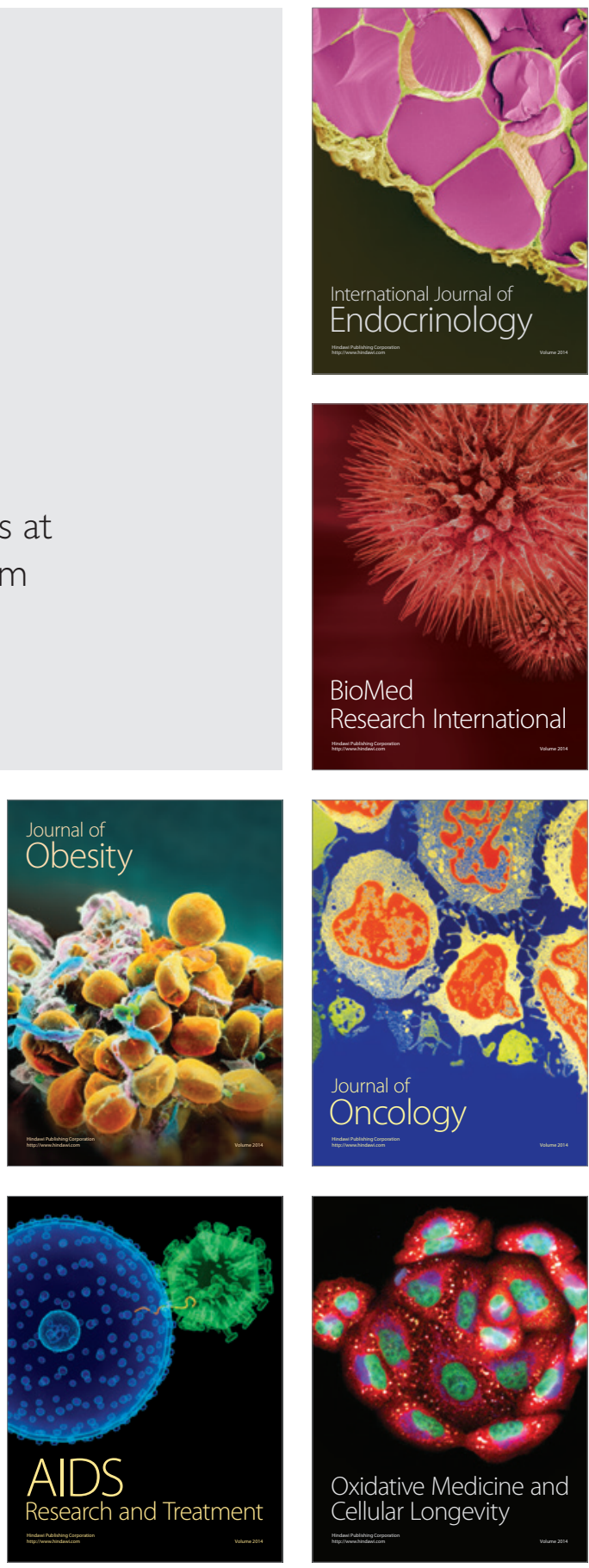